\section{Enforcement of the food hygiene package: role and requirements from a recent European Union Court's judgement}

\author{
Claudio Biglia, ${ }^{1}$ Daniele Pisanello \\ 'Dipartimento di Sanità Pubblica,
}

Azienda Sanitaria Locale Torino 1, Torino; ${ }^{2}$ Libero professionista, Avvocato consulente in Diritto Alimentare, Lecce, Italy

\section{Abstract}

Detection of non compliance with hygienic requirements is the major issue of a recent judgement of the Court of Justice of the European Union (CJEU). As long as the hygiene package addresses some obligation under condition as where necessary, if appropriate and similar, it is up to the official control to identify whether a infraction is at stake. In October 2011, the CJEU established the need of a correlation between the requirements regulated in the Annex of Regulation (EC) No. 852/2004 and Article 4(2) of the same regulation on one side, and the context of those provisions, on the other. By this way, the Court affirms that food business operators have to put adopt, implement and maintain a permanent procedure or procedures based on the Hazard Analysis Critical Control Point (HACCP) principles. Since hygienic requirements must be interpreted so as not to deprive Article 5 of the Reg. (EC) No. 852/2004 of effectiveness, it follows that when competent Authorities seemingly do not reckon actual contamination, one cannot conclude that a breach to law occurred on the basis of the finding that a potential purchaser could conceivably have touched foodstuff by hand or sneezed on it only. In such a case, competent Authority should take into consideration measures taken by those operators under Article 5 of the regulation in order to prevent, eliminate or reduce to acceptable levels the hazard inherent to the food process.

This statement is of crucial importance since it requires a stronger effort by the official control in detecting violations to food law and gives room to a number of perspective shortcomings between EU food hygiene law and the Italian criminal food law.

\section{Introduzione}

L'entrata in vigore del cosiddetto (c.d.) pacchetto igiene ha comportato una rivoluzione copernicana di vasto momento per le autorità del controllo ufficiale degli alimenti e mangimi. L'adeguamento delle strutture e delle procedure che, a livello locale, devono dare attuazione ed esecuzione ai principi e regole scolpiti nei regolamenti sull'igiene è operazione ancora in itinere, in Italia e, come denota il caso giurisprudenziale qui commentato, anche all'estero. Tra gli aspetti maggiormente controversi vi è certamente il frequente ricorso del dato normativo a requisiti elastici da parte della disciplina igienico-sanitaria di fonte comunitaria. Il frequente impiego, specie negli allegati tecnici dei regolamenti sull'igiene, di termini quali ove necessario, ove opportuno, adeguato 0 sufficiente gioca un ruolo decisivo nel marcare il limes tra conforme e non conforme e, di riflesso, nel bilanciamento dei diritti e obblighi sia del controllo ufficiale che dell'operatore del settore alimentare (OSA).

In questa prospettiva la sentenza del 6 ottobre 2011, pronunciata dalla Corte di Giustizia nella causa C-382/2010 (d'ora in avanti, per brevità anche sentenza Albrecht) assume una rilevanza ben più ampia della fattispecie concreta da cui ha tratto origine. Con essa la Corte ha tracciato con chiarezza alcune modalità operative alle quali il controllo ufficiale dovrà attenersi nel valutare la sussistenza di una violazione ai requisiti igienici di cui al pacchetto igiene.

\section{II pacchetto igiene e i requisiti elastici: il dato normativo}

Come noto, l'adozione del c.d. pacchetto igiene, l'insieme dei regolamenti e direttive comunitarie che hanno riscritto le regole armonizzate sull'igiene dei prodotti alimentari e il relativo controllo ufficiale, è stata propiziata dal Regolamento (CE) No 178/2002 (Parlamento Europeo, 2002), l'atto istitutivo di un compiuto sistema giuridico di legislazione alimentare nei 27 paesi dell'Unione Europea (UE).

La nuova food policy europea, tesa a una rimodulazione delle competenze, con una diarchia tra valutatori e gestori del rischio, e concentrazione a livello comunitario delle competenze decisorie, con contestuale sottrazione di porzioni di sovranità agli stati membri, ha portato nel settore della legislazione igienicosanitaria una ridefinizione delle norme generali in materia di igiene dei prodotti alimentari. L'adozione del c.d. pacchetto igiene, infatti, non è stata una mera compilazione di norme già predisposte da direttive risalenti agli anni ' 80 e ' 90 , bensì ha rappresentato la maturazione di un processo di riformulazione della disciplina igienico-sanitaria sulla base di nuovi principi ispiratori maturati a livello internazionale (ad esempio la Commissione del Codex Alimentarius). Una lettura coordinata del Reg.
Correspondence: Claudio Biglia, Dipartimento di Sanità Pubblica, Azienda Sanitaria Locale - ASL Torino 1, corso Vigevano 46, 10100 Torino, Italy. Tel. +39.011 .70958869 - Fax: +39.011 .70958880 . E-mail: big_c@libero.it

Key words: Official control, Food contamination, Food business operator, Hygiene, Food law.

Conflict of interests: the authors declare no potential conflict of interests.

Received for publication: 15 January 2013. Revision received: 16 July 2013.

Accepted for publication: 16 July 2013.

This work is licensed under a Creative Commons Attribution 3.0 License (by-nc 3.0).

(C) Copyright C. Biglia and D. Pisanello, 2013 Licensee PAGEPress, Italy

Italian Journal of Food Safety 2013; 2:e42 doi:10.4081/ijfs.2013.e42

(CE) No 178/2002 (Parlamento Europeo, 2002) e del c.d. pacchetto igiene ha dimostrato (Pisanello et al., 2010) che il rapporto tra impresa alimentare e il sorvegliante pubblico risulta imperniato su due elementi fondamentali: i) la responsabilità piena dell'operatore privato per la conformità alla legislazione alimentare del prodotto e del processo produttivo, da un lato, e ii) i poteri del controllo ufficiale a fronte della flessibilità delle prescrizioni igienico-sanitarie vigenti, dall'altro.

Più in generale la disciplina igienico-sanitaria è incardinata sui seguenti principi: i) la responsabilità principale per la sicurezza degli alimenti incombe all'operatore del settore alimentare; ii) garanzia della sicurezza degli alimenti lungo tutta la catena alimentare, a cominciare dalla produzione primaria; iii) mantenimento della catena del freddo per gli alimenti che non possono essere immagazzinati a temperatura ambiente in condizioni di sicurezza, in particolare per quelli congelati; iv) l'applicazione generalizzata di procedure basate sui principi del sistema Hazard Analysis Critical Control Point (HACCP), unitamente all'applicazione di una corretta prassi igienica, dovrebbe accrescere la responsabilità degli operatori del settore alimentare; v) i manuali di corretta prassi costituiscono uno strumento prezioso per aiutare gli operatori del settore alimentare nell'osservanza delle norme d'igiene a tutti i livelli della catena alimentare e nell'applicazione dei principi del sistema HACCP; vi) determinazione dei criteri microbiologici e requisiti in materia di controllo delle temperature sulla base di una valutazione scientifica dei rischi.

Il punto nodale di questa disciplina e, al contempo, la sfida lanciata sia all'autorità pubblica sia all'operatore privato, riposa esattamente 
nel modo di attuazione di quelle clausole quali ove opportuno, se necessario, qualora necessario, per quanto ragionevolmente possibile che caratterizzano molte delle disposizioni dei Regolamenti (CE) nn. 852/2004 (Parlamento Europeo, 2004c) e 853/2004 (Parlamento Europeo, 2004a). Lo stesso art. 4.3 del Reg. (CE) No. 852/2004 è latore di tale flessibilità. Sul punto, come noto, valgono le precisazioni di cui alle Linee guida sull'applicazione di alcune disposizioni del Regolamento (CE) No 852/2004 relativo all'igiene dei prodotti alimentari (Commissione europea, 2009).

Una tecnica legislativa di tal genere segna il cambiamento della politica alimentare comunitaria: alla ferrea e spesso dettagliata disciplina, propria delle direttive previgenti, si è preferita una regolamentazione elastica che lasci all'operatore privato il compito - e le connesse responsabilità - di individuare ed applicare soluzioni produttive e organizzative adatte alla propria realtà aziendale, tali, in ogni caso, da garantire il livello elevato di sicurezza alimentare.

Applicandosi i regolamenti del c.d. pacchetto igiene, e il connesso grado di flessibilità, il limes tra il conforme e il non conforme è condizionato da un numero di fattori tra i quali speciale importanza hanno i seguenti: la realtà concreta relativa all'unità produttiva (grado di rispetto dei requisiti igienici e delle prestazioni dei processi anche organizzativi), la percezione dell'impresa (o di una porzione di essa) da parte dell'autorità compente del Reg. (CE) No. 882/2004 (Parlamento Europeo, 2004b).

Entrambi i fattori, prestazioni rese e percezione da parte dell'autorità competente, sono dei fattori condizionabili - con l'adeguato know how legale e tecnico - da parte dell'impresa in vista di ispezione, controllo, verifica 0 audit ai sensi del Reg. (CE) No 882/2004.

\section{Case Report}

La causa decisa con la sentenza del 6 ottobre 2011 della Corte dell'UE verte esattamente sui criteri mediante i quali l'autorità competente può, legittimamente, contestare una violazione ai requisiti igienici di cui al Reg. (CE) No 852/2004 (Parlamento Europeo, 2004c).

Una breve illustrazione del caso concreto aiuterà la comprensione della sentenza. Alcuni operatori commerciali della distribuzione alimentare operanti in Austria, adivano il giudice per reclamare contro alcune misure restrittive e sanzionatorie che l'Autorità competente aveva intrapreso nei confronti della vendita di prodotti da forno mediante contenitori self service.

A seguito di controlli ufficiali, condotti sotto l'egida del Reg. (CE) No 882/2004 (Parlamento
Europeo, 2004b), l'autorità competente aveva verificato che erano stati predisposti contenitori destinati alla vendita in self service di prodotti da forno i cui coperchi erano provvisti di manici che consentivano di sollevarli con una mano, mentre, con l'altra, potevano essere presi i prodotti mediante una pinza messa a disposizione del cliente. In seguito, quest'ultimo doveva rimettere la pinza al suo posto e richiudere il coperchio. Rispetto a tale evidenza, l'autorità competente austriaca aveva imposto a tali operatori di allestire contenitori destinati alla vendita di tali prodotti in self service in modo tale che questi ultimi potessero essere presi solo con l'ausilio di strumenti tecnici, come pinze o dispositivi di erogazione, $\mathrm{e}$ che non fosse possibile la reintroduzione di prodotti da forno già prelevati. Ad avviso dell'autorità di controllo, infatti, tali contenitori per la vendita in self service presentavano l'inconveniente di consentire ai clienti di prendere e toccare le merci a mani nude, nonché di tossire e starnutire su dette merci; inoltre, era stigmatizzato il fatto che il dispositivo di chiusura non impedisse al cliente di reintrodurre la merce nel contenitore e l'assenza di protezione dei prodotti alimentari agli starnuti dei clienti potesse provocare il deposito, su tali prodotti, di germi e virus.

$\mathrm{Su}$ questi rilievi, l'autorità di controllo accertava una violazione del punto 3 del capitolo IX dell'allegato II del Reg. (CE) No 852/2004 (Parlamento Europeo, 2004c) in forza del quale: In tutte le fasi di produzione, trasformazione e distribuzione gli alimenti devono essere protetti da qualsiasi forma di contaminazione atta a renderli inadatti al consumo umano, nocivi per la salute o contaminati in modo tale da non poter essere ragionevolmente consumati in tali condizioni.

Il giudice, adito per appurare la legittimità dei provvedimenti della pubblica autorità, considerando che la soluzione delle controversie sottopostegli richiedessero l'interpretazione dell'allegato II, capitolo IX, punto 3, del Reg. (CE) No 852/2004 (Parlamento Europeo, 2004c), sospendeva il procedimento e sottoponeva alla Corte le seguenti questioni pregiudiziali: i) In base a quali criteri si deve accertare se un prodotto alimentare sia inadatto al consumo umano ai sensi dell'allegato II, capitolo IX, punto 3, del [regolamento]. Se, a tal fine, sia sufficiente che un potenziale acquirente possa aver toccato un alimento in vendita o averci starnutito sopra; ii) in base a quali criteri si deve accertare se un prodotto sia nocivo per la salute ai sensi dell'allegato II, capitolo IX, punto 3, del [regolamento]. Se, a tal fine, sia sufficiente che un potenziale acquirente possa aver toccato un alimento in vendita o averci starnutito sopra; iii) in base a quali criteri si deve accertare se sussista una contaminazione tale che non si possa più ragionevolmente considerare che un alimento possa essere consuma- to nelle condizioni in cui si trova ai sensi dell'allegato II, capitolo IX, punto 3, del Iregolamento]. Se, a tal fine, sia sufficiente che un potenziale acquirente possa aver toccato un alimento in vendita o averci starnutito sopra.

\section{Discussione}

Come si vede, la disposizione tecnica contestata e, conseguentemente, la formulazione dei quesiti formulati alla Corte, vertono su tre nozioni centrali per la legislazione alimentare: alimento inadatto, alimento nocivo, alimento contaminato.

Se si eccettua il caso dell'alimento contaminato, per il quale è dato individuare nel Reg. (CE) No 852/2004 (Parlamento Europeo, 2004c) una definizione specifica [la «contaminazione» è infatti definita come la presenza o l'introduzione di un pericolo dall'art. 2.1 (f) Reg. (CE) No 852/2004], i concetti di alimento nocivo e inadatto non sono normati dal regolamento sull'igiene; stante però il rinvio operato da questo regolamento alle definizioni del Reg. (CE) No 178/2002 (Parlamento Europeo, 2002) $\mathrm{e}$, più in generale, viste le interrelazioni tra $\mathrm{i}$ due atti normativi, è a quest'ultimo che potrebbe ricorrersi per dare contenuto definitorio alle nozioni portate in causa. Nel Reg. (CE) No 178/2002, infatti, si trova una disposizione, l'articolo 14, interamente dedicata agli alimenti a rischio i quali, secondo il Reg. (CE) No 178/2002 danno luogo ai casi seguenti: i) alimenti dannosi per la salute, e ii) alimenti inadatti al consumo umano.

Il Reg. (CE) No 178/2002 (Parlamento Europeo, 2002), all'art. 14.4 specifica la nozione di alimento dannoso e d'alimento inadatto. Quanto al primo, in una prospettiva decisamente ampia, il regolamento quadro stabilisce che per determinare se un alimento sia dannoso per la salute occorre prendere in considerazione quanto segue: i) i probabili effetti immediati e/o breve termine, e/o lungo termine dell'alimento sulla salute di una persona che lo consuma, ma anche su quella dei discendenti; ii) i probabili effetti tossici cumulativi di un alimento; iii) la particolare sensibilità, sotto il profilo della salute di una specifica categoria di consumatori nel caso in cui l'alimento sia destinato ad essa. Quanto all'alimento inadeguato all'uso umano, il Reg. (CE) No 178/2002 (Parlamento Europeo, 2002) riferisce tale caso alla contaminazione dovuta a materiale estraneo o a altri motivi, o in seguito a putrefazione, deterioramento o decomposizione [art. $14.5 \mathrm{del}$ Reg. (CE) No 178/2002].

Sarebbe stato oltremodo utile che la Corte di Giustizia dell'Unione Europea (CGUE) si pronunciasse sui criteri per determinare l'inaccettabilità del rischio alimentare. In realtà la CGUE glissa completamente sui collegamenti 
tra Reg. (CE) No 178/2002 e il regolamento sull'igiene, preferendo riformulare i quesiti in un'ottica leggermente diversa: il giudice del rinvio chiede, sostanzialmente, se l'allegato II, capitolo IX, punto 3, del regolamento debba essere interpretato nel senso che, in circostanze come quelle oggetto della causa principale, nel caso di contenitori destinati alla vendita in self service di prodotti da forno, il fatto che un potenziale acquirente possa aver teoricamente toccato a mani nude gli alimenti in vendita o starnutito su questi ultimi consenta, di per sé, di constatare che tali alimenti non sono stati protetti da qualsiasi forma di contaminazione atta a renderli inadatti al consumo umano, nocivi per la salute o contaminati in modo tale da non poter essere ragionevolmente consumati in tali condizioni (cfr. par. 15, sentenza Albrecht).

L'accorpamento delle tre distinte questioni risulta funzionale alla strategia decisoria della Corte la quale, circoscrivendo il perimetro della decisione interamente all'interno del Reg. (CE) No 852/2004 (Parlamento Europeo, 2004c), apre la via ad una scelta tra una nozione astratta di contaminazione contrapposta a una nozione concreta.

Il ragionamento sviluppato dalla CGUE parte dal riconoscere che la disposizione impugnata [il punto 3, capitolo IX, allegato II del Reg. (CE) No 852/2004] è relativo all'obbligo di protezione dell'alimento da qualsiasi forma di contaminazione atta a renderli inadatti al consumo umano, nocivi per la salute o contaminati in modo tale da non poter essere ragionevolmente consumati in tali condizioni. La CGUE ha buon gioco nel sottolineare che questo è un requisito che gli operatori del settore alimentare devono rispettare in forza dell'art. $4, \mathrm{n} .2$ dello stesso regolamento ai sensi del quale Gli operatori del settore alimentare che eseguono qualsivoglia fase della produzione, della trasformazione e della distribuzione di alimenti successiva a quelle di cui al paragrafo 1 , rispettano $i$ requisiti generali in materia d'igiene di cui all'allegato II e ogni requisito specifico previsto dal regolamento (CE) n. 853/2004 (Parlamento Europeo, 2004a).

Su questo dato normativo s'innesta l'elemento fondamentale posto dalla sentenza Albrecht: le norme ora citate devono essere applicate e, se del caso, interpretate alla luce del contesto in cui si collocano secondo la consolidata giurisprudenza della CGUE (così, testualmente, par. 18 della sentenza Albrecht). L'aver posto l'accento sull'imprescindibilità di un'applicazione e interpretazione sistematica dei requisiti igienici consente alla CGUE di stabilire un collegamento giuridico stringente coll'art. 5 del Reg. (CE) No 852/2004 (Parlamento Europeo, 2004c) che, come noto, è relativo all'obbligo di autocontrollo igienico dell'industria alimentare in base ai principi dell'HACCP. Interessante osservare che questo collegamento era stato caldeggiato nelle memorie presentate dal governo ceco e da quello dei Paesi Bassi, nonché dalla Commissione europea (si veda par. 18, sentenza Albrecht).

Ai sensi del citato art. 5 numero 1, gli operatori del settore alimentare predispongono, attuano e mantengono una 0 più procedure permanenti basate sui principi del sistema HACCP. La CGUE esplicita, tra tali principi, quello contenuto nell'art. 5, n. 2, lett. a), del regolamento, il quale impone di identificare ogni pericolo che deve essere prevenuto, eliminato 0 ridotto a livelli accettabili. La previsione di un obbligo di predisposizione, attuazione e aggiornamento di procedure d'autocontrollo igienico è, nel ragionamento della CGUE, espressione dello scopo perseguito dal legislatore dell'Unione di attribuire la responsabilità principale in materia di sicurezza degli alimenti agli operatori del settore alimentare (cfr. par. 20 , sentenza Albrecht) ed è a tale principio che l'allegato II, capitolo IX, punto 3, del regolamento deve accordarsi, pertanto, essere interpretato in modo da non privare l'art. 5 di tale regolamento del suo effetto utile (cfr. par. 21 sentenza Albrecht).

Nel caso di specie, la sola interpretazione utile a salvaguardare l'attribuzione di responsabilità all'OSA (e il senso dei costi connessi) risiede in ciò che la violazione dell'obbligo di protezione degli alimenti dal rischio di contaminazione deve essere investigata e valutata in rispetto alle procedure di autocontrollo igienico predisposte dall'operatore privato. La valutazione del rispetto dei requisiti igienici deve quindi essere svolta anche e, sembrerebbe, soprattutto alla luce delle procedure di autocontrollo (cfr. par. 22 sentenza Albrecht).

Tale valutazione deve essere condotta, afferma la Corte, in termini scientifici, discutendo con l'OSA: Ne consegue che, in una circostanza come quella oggetto della causa principale, dalla quale non emerge che un'effettiva contaminazione sia stata rilevata dalle autorità competenti, non si può concludere che gli operatori del settore alimentare interessati abbiano violato tale punto 3 sulla base della sola constatazione che un potenziale acquirente possa aver teoricamente toccato a mani nude gli alimenti in vendita o starnutito su questi ultimi, senza prendere in considerazione le misure che tali operatori hanno adottato conformemente all'art. 5 del regolamento al fine di prevenire, eliminare o ridurre ad un livello accettabile il rischio che può presentare una contaminazione ai sensi dell'allegato II, capitolo IX, punto $3, d i$ tale regolamento e senza contestare l'insufficienza delle misure adottate a tale proposito sulla base di tutti $i$ dati pertinenti disponibili (cfr. par. 22, sentenza Albrecht). Nel caso di specie, peraltro, tutti i pareri tecnici escludevano la sussistenza di problemi igienici nell'uso dei contenitori controversi.

\section{Conclusioni}

La sentenza Albrecht è tale da poter mettere in moto un processo di revisione delle modalità di esecuzione del controllo ufficiale che siano maggiormente confacenti agli indirizzi di food policy vigenti nel mercato unico europeo. Certamente, d'ora in avanti, la mera ricognizione di una mancata osservanza a uno dei requisiti igienici non sarà sufficiente a integrare una violazione giuridicamente rilevante: la contrarietà alle norme comunitarie, sembra potersi desumere dalla sentenza, abbisognerà di un quid pluris e cioè di una valutazione, basata sull'analisi del pericolo, nel contesto delle procedure igieniche predisposte e attuate dall'OSA. Il che evidentemente chiama l'operatore del controllo ufficiale a un maggiore sforzo di valutazione delle procedure e delle realtà sottoposte a controllo, rifuggendo una pratica investigativa meramente formale e ottusamente limitata a requisiti considerati in sé e per sé.

Una tale lettura che, come detto, sembra essere il portato principale della sentenza Albrecht, è dunque funzionale all'accrescimento della sensibilità dell'OSA rispetto alle procedure igieniche spesso avvertite come un mero costo. A voler poi ragionare con i termini sanzionatori del decreto legislativo n. 193/2007 (Repubblica Italiana, 2007) si potrebbe dire che, in un caso come quello della causa principale, non sembrerebbe corretto procedere con una contestazione ex articolo 6, comma 5, per mancato rispetto dei requisiti igienici in quanto, in assenza di ulteriori evidenze, non vi sarebbe violazione giuridicamente rilevante, stando alla sentenza Albrecht. Semmai, sembrerebbe più congruo, eventualmente, procedere alla contestazione dell'articolo 6 , comma 7 , che consente un periodo di adeguamento.

In ogni caso, sia che si proceda in termini esclusivamente sanzionatori 0 anche prescrittivi [come stabilito da art. 54 del Reg. (CE) No 852/2004 (Parlamento Europeo, 2004c)] il fulcro riposa sulla giustificazione scientifica: l'inadeguatezza del prodotto, del processo, della procedura dovrà essere sempre adeguatamente motivato dall'autorità di controllo. Obbligo di motivazione peraltro già operativo nel nostro sistema amministrativo, giusto l'articolo 2 della legge No 241/1990 (Repubblica Italiana, 1990). La sentenza Albrecht, se adeguatamente valorizzata e studiata, potrebbe contribuire altresì ad aggiornare i rapporti tra la disciplina igienica degli alimenti di matrice comunitaria e la legge penale alimentare di parte speciale, in particolare la contravvenzione del cattivo stato di conservazione.

Se, come e quando i richiami della CGUE saranno recepiti dalla giurisprudenza italiana in tema di articolo 5, lett. (b), Legge No 283/1962 (Repubblica Italiana, 1962), essi 
sono i tre ambiti di riflessione sui quali occorrerebbe investire in modo interdisciplinare.

\section{Bibliografia}

Commissione europea, 2009. Linee guida sull'applicazione di alcune disposizioni del Regolamento (CE) No 852/2004 relativo all'igiene dei prodotti alimentari, 16 febbraio 2009, SANC0/1731/2008 Rev. 6. Commissione Europea ed., Bruxelles. Disponibile al sito: http://bdr.rete.basilicata.it/c/document_library/get_file?p_l_id=1 $7192 \&$ folderId $=15846 \&$ name $=$ DLFE 679.pdf

Parlamento Europeo, 2002. Regolamento del Parlamento europeo e del Consiglio, del 28 gennaio 2002, che stabilisce i principi e i requisiti generali della legislazione alimentare, istituisce l'Autorità europea per la sicurezza alimentare e fissa procedure nel campo della sicurezza alimentare, 178/2002/CE. In: Gazzetta Ufficiale, L 31/1,
01/02/2002.

Parlamento Europeo, 2004a. Regolamento del Parlamento europeo e del Consiglio, del 29 aprile 2004, che stabilisce norme specifiche in materia di igiene per gli alimenti di origine animale, 853/2004/CE. In: Gazzetta Ufficiale, L 139/55, 30/4/2004.

Parlamento Europeo, 2004b. Regolamento del Parlamento europeo e del Consiglio, del 29 aprile 2004, relativo ai controlli ufficiali intesi a verificare la conformità alla normativa in materia di mangimi e di alimenti e alle norme sulla salute e sul benessere degli animali, 882/2004/CE. In: Gazzetta Ufficiale, L 191, 28/05/2004.

Parlamento Europeo, 2004c. Regolamento del Parlamento europeo e del Consiglio, del 29 aprile 2004, sull'igiene dei prodotti alimentari, 852/2004/CE. In: Gazzetta Ufficiale, L 139/1, 30/4/2004.

Pisanello D, Biglia C, Pellicano CM, 2010. Guida alla legislazione alimentare. L'applicazione pratica, il controllo ufficiale, l'audit, le responsabilità, le sanzioni, l'etichettatura, la pubblicità. EPC Libri,
Roma.

Repubblica Italiana, 1962. Decreto legislativo del 30 aprile 1962, n. 283. Modifica degli artt. 242, 243, 247, 250 e 262 del t.u. delle leggi sanitarie approvato con r.d. 27 luglio 1934, n. 1265. Disciplina igienica della produzione e della vendita delle sostanze alimentari e delle bevande. In: Gazzetta Ufficiale n. 139, 04/06/1962.

Repubblica Italiana, 1990. Decreto legislativo del 7 agosto 1990, n. 241. Nuove norme in materia di procedimento amministrativo e di diritto di accesso ai documenti amministrativi. In: Gazzetta Ufficiale n. 192, 18/08/ 1990.

Repubblica Italiana, 2007. Decreto legislativo del 6 novembre 2007, n. 193. Attuazione della direttiva 2004/41/CE relativa ai controlli in materia di sicurezza alimentare e applicazione dei regolamenti comunitari nel medesimo settore. In: Gazzetta Ufficiale n. 261 - Suppl. Ordinario n. 228, 09/11/2007. 